\title{
Der Schottenzug durch das norwegische Gudbrandsdal im Jahre 1612. Eine Episode am Rande des Kalmarer Krieges von 1611 bis 1613
}

Im Kriegsgeschichtlichen Museum (Gudbrandsdal krigsminnesamling) in der Ortschaft Kvam, 90 km nördlich von Lillehammer, mitten im norwegischen Gudbrandsdal, befindet sich eine Tafel mit folgender Inschrift:

"Epitaphium: Her under hviler Her. Oberste Georg Jörgen Sinkler som falt i Kringlene Aaret 1612. med et antal af 900. Skaatter som blev knust som leerpotter. af et mindre Tal af 300 Bönder af Læsjö, Waage, Froen. og den Böndernes Andförer, det var Berdon Sejelstad, af Ringeboe Sogn ${ }^{1}$.«

Lesen wir diesen Text, so fallen uns sofort zwei Zahlen ins Auge, nämlich die angeblich von weniger als 300 norwegischen Bauern geschlagenen und vernichteten 900 schottischen Söldner. Hier sind wir schon mittendrin in der Legenden- und Sagenbildung, die den Schottenzug ${ }^{2}$ bis heute zu einem wichtigen Bestandteil der Regionalgeschichte des Gudbrandsdals ${ }^{3}$, wenn nicht sogar der norwegischen Nationalgeschichte gemacht hat, denn die auf der Grabinschrift genannten Zahlen sind falsch. Das Zahlenverhältnis der Kombattanten war eher umgekehrt, wie noch zu sehen sein wird.

In diesem Beitrag möchte ich die überprüfbaren Tatsachen zum Schottenzug im historischen Kontext darstellen sowie die Entstehung der vielen Legenden und Sagen rund um den Schottenzug herausarbeiten.

Dabei werde ich in vier Schritten vorgehen. Zunächst wende ich mich der Quellenlage $\mathrm{zu}$, um mich anschließend dem historischen Kontext $\mathrm{zu}$ widmen. Im dritten Schritt gehe ich auf den Schottenzug selbst ein und schließlich beschäftige ich mich mit der Legenden- und Sagenbildung rund um den Schottenzug.

1 "Grabschrift: Hier ruht der Herr Oberst Georg Jörgen Sinkler (George Sinclair), der im Jahre 1612 bei Kringelen (heute: Kringen) fiel. Mit 900 Schotten, die wie Tontöpfe von weniger als 300 Bauern aus Læsjø (heute: Lesja), Waage (heute: Vågå) und Froen (heute: Fron) vernichtet wurden. Und ihr Anführer war Berdon Sejelstad aus dem Kirchspiel Ringebu.« - Alle Übersetzungen aus dem Norwegischen wurden vom Verfasser vorgenommen.

2 Wird in Norwegen vom "skottetog " gesprochen, so wissen sofort alle historisch Interessierten, insbesondere im Gudbrandsdal, welches Ereignis gemeint ist. In diesem Aufsatz werde ich die deutsche Übersetzung "Schottenzug « verwenden.

3 Das Tal erstreckt von sich Lillehammer $200 \mathrm{~km}$ in Richtung Nordwesten. Zum Gudbrandsdal - ich bevorzuge hier die norwegische Schreibweise - werden auch weiter westlich gelegene Seitentäler wie das Ottadal, das Sjodal, das Heidal, das Espedal und das Gausdal gerechnet. 


\section{Zur Quellenlage}

Die wichtigste norwegische Quelle zum Schottenzug stammt vom dänischen Statthalter auf der Festung Akershus, Enevold Kruse, der am 17. September 1612, also nur gut drei Wochen nach den blutigen Ereignissen im Gudbrandsdal, einen ausführlichen Bericht nach Kopenhagen sandte ${ }^{4}$. Ein weiterer Bericht des Enevold Kruse über den Schottenzug, datiert vom 3 . Oktober 1612, wurde später im dänischen Reichsarchiv in Kopenhagen gefunden und in Norwegen zum ersten Mal im Jahre 1877 veröffentlicht ${ }^{5}$. Dieser zweite Bericht des Enevold Kruse wiederholt im wesentlichen das, was er schon im ersten nach Kopenhagen gemeldet hatte.

Wichtige Quellen, die die Darstellungen des Enevold Kruse untermauern, sind drei Dank- und Schenkungsurkunden, alle datiert vom 3. September 1613. Hierin dankte der dänische König den Anführern des norwegischen Bauernheeres, Lars Hage, Peder Randklev und Berdon Sejelstad für ihren Einsatz und ihre Tapferkeit beim Kampf gegen die schottischen Söldner und überließ ihnen als Anerkennung drei Höfe aus seinen Krongütern ${ }^{6}$. Waren die Genannten aber wirklich so tapfer? Auch diese Frage wird zu untersuchen sein.

Auf englischer bzw. schottischer Seite existieren ebenfalls einige Quellen zum Thema. Im Public Record Office in London enthält zum Beispiel die Korrespondenz des englischen Königs Jacob I. mit Sir Robert Anstruther, seinem Gesandten am dänischen Hof, wichtige Hinweise auf die Anwerbung schottischer Söldner für Schweden sowie über den Schottenzug?

Im Staatsarchiv in Stockholm befindet sich neben anderen eine weitere interessante Quelle, nämlich ein Brief Gustav II. Adolf, datiert vom 16. November 1611, an den schottischen Offizier Sir James Spens mit der Bitte, bis zum 1. April 1612 3000 Infanteriesoldaten für den Krieg gegen Dänemark anzuwerben ${ }^{8}$. Doch nicht nur dieser ausländische Offizier wurde mit der Anwerbung von Söldnern beauftragt, wie noch zu sehen sein wird.

Wollen wir die wirklichen Ereignisse rund um den Schottenzug rekonstruieren, dann müssen wir uns auf die bekannten, hier dargestellten Quellen beschränken. Auf norwegischer Seite haben sich aber im Laufe der Jahrhunderte Sagen und Legenden gebildet, die bis heute unkritisch überliefert werden, aber einer quellenkritischen Untersuchung nicht standhalten.

4 Der Bericht von Enevold Kruse ist wiedergegeben bei Thomas Michell, History of the Scottish Expedition to Norway, 1612, Edinburgh 1886; im Jahre 1997 von Jon Selfors unter dem norwegischen Titel »Thomas Michell, Historien om den skotske Ekspedisjon til Norge i 1612«, o.O. 1997 herausgegeben, siehe hier S. 206-208. Thomas Michell war englischer Generalkonsul in Norwegen. Auf einer Reise durch das Gudbrandsdal im Herbst 1884 hörte er zum ersten Mal vom Schottenzug. Von den wenigen, die bislang versucht haben, den Schottenzug quellenkritisch zu analysieren, war Michell der erste.

5 Olaf Olafsen, Skottetoget efter Folkesagnet og Historien, Molde 1877.

6 Michell, Historien (wie Anm. 4), S. 15. Siehe auch Ragnar Øvrelid, Skottetoget 1612, in: Gudbrandsdal krigsminnesamling Årsskrift 1997, S. 13.

7 . Teile der Korrespondenz sind abgedruckt bei Michell, Historien (wie Anm. 4), S. 159-168.

8 Der Brief ist in lateinischer Sprache sowie in der englischen Übersetzung abgedruckt ebd., S. 199-203. 


\section{Der historische Kontext des Schottenzuges}

Den historischen Hintergrund für den Schottenzug durch das Gudbrandsdal bildet der Kalmarer Krieg von 1611 bis 1613 zwischen Schweden auf der einen und Dänemark-Norwegen auf der anderen Seite?

Unter der Herrschaft Erik XIV. (1560-1568) aus dem Hause Wasa begann Schwedens Kampf um die Vorherrschaft im Ostseeraum ${ }^{10}$. Einer der wichtigsten Rivalen Schwedens war Dänemark. Das Ziel, nämlich die Vorherrschaft im Ostseeraum zu erlangen, sollte fast 250 Jahre ein Hauptmotiv der schwedischen Außenpolitik sein. Erster Erfolg dieser Politik war zweifelsohne der Erwerb Estlands im Jahre 1561. Jetzt war Schweden imstande, große Teile des Handels mit Rußland zu kontrollieren. Schwedens Expansionsdrang nach Osten sowie seine gleichzeitigen Bemühungen, die politischen und handelspolitischen Beziehungen zu Westeuropa auszubauen, riefen in Dänemark starke Unruhe hervor, sah man doch die eigene Position in Nordeuropa bedroht. Im folgenden Siebenjährigen Nordischen Krieg (1563-1570) konnte sich Schweden nur mit Mühe gegen Dänemark behaupten, und im Frieden von Stettin wurde Schweden gezwungen, ein respektables Lösegeld für die an der schwedischen Westküste am Göta-Fluß gelegene Festung Älvsborg zu zahlen, die von dänischen Truppen besetzt worden war ${ }^{11}$.

Allerdings bildeten auch in den folgenden Jahrzehnten insbesondere handelspolitsche Rivalitäten zwischen Schweden und Dänemark-Norwegen die Ursache für weitere Spannungen. Schweden beherrschte jetzt den Finnischen Meerbusen und den Seeweg ins Baltikum, was zu empfindlichen Einschränkungen der dänischen Schiffahrt in dieser Region führte. Weiter ging es um den Öresundzoll, der für den Beherrscher des Sundes eine beträchtliche Einnahmequelle darstellte. Schließlich schickte sich Schweden an, zum Nordkap und zum Eismeer vorzustoßen, um so die Kontrolle über die Handelsverbindungen von Westeuropa rund um das Nordkap nach Rußland zu erlangen ${ }^{12}$. Auf einen Nenner gebracht, ging es um die politische und handelspolitische Vorherrschaft im gesamten skandinavischen Raum.

Der dänische König Christian IV. hatte weitreichende militärische Pläne. Er selbst beabsichtigte, an der Spitze einer Heeresabteilung von Blekinge aus auf die Feste Kalmar vorzustoßen. Eine weitere Abteilung sollte von Halland aus gegen Jönköping vorrücken, während ein norwegisches Bauernheer von 6000 Soldaten von Båhuslen und Østfold aus ins Götaland einfallen sollte. Über diese Pläne hat-

9. Im Jahre 1536 hatte Norwegen seine gleichberechtigte Stellung in der dänisch-norwegischen Union verloren und war Teil des dänischen Königreiches geworden. Der Landesteil Norwegen wurde von einem vom dänischen König eingesetzten Statthalter von der Festung Akershus aus verwaltet und regiert. Siehe auch: Norges Historie. Red.: Knut Mykland, Bd 5: Fra rike til provins 1448-1536, 3. oppl., Oslo 1992, S. 437 f.

10 Zum politischen Hintergrund des Kalmarer Krieges siehe Arthur Erwin Imhof, Grundzüge der nordischen Geschichte, Darmstadt 1970, S. 99-103; Norges Historie. Red.: Knut Mykland, Bd 6: Gjenreisning 1536-1648, Oslo 1992, S. 364-339; Jörgen Weibull, Schwedische Geschichte, Stockholm 1993, S.42-44.

11 Weibull, Schwedische Geschichte (wie Anm. 10), S. 42.

12 Norges Historie, Bd 6 (wie Anm. 10), S. 363. Der schwedische König Karl IX. hatte Göteburger Bürgern Handelsprivilegien für das Gebiet Varanger-Tysfjord in der heutigen Nord-Finnmark ausgestellt. 
te Christian IV. seinen Statthalter auf der Festung Akershus, Enevold Kruse, am 3. März 1611 informiert ${ }^{13}$. Allerdings war König Christian IV. genötigt, mitteleuropäische Söldner anzuwerben, um seine ambitiösen Kriegspläne verwirklichen zu können. Schon vor der offiziellen Kriegserklärung war die Werbung dieser Söldner in vollem Gang. Schließlich kämpften mehrere tausend deutsche, französische und englische Söldner auf dänischer Seite. Einige Forscher sprechen von bis zu 18000 europäischen Söldnern ${ }^{14}$.

Wichtige strategische Ziele der dänischen Truppen waren die schwedischen Festungen Kalmar und erneut Älvsborg. Allerdings verlief der dänische Feldzug nicht nach Plan. Die Versorgung der Truppen bereitete Schwierigkeiten, und die daraus resultierenden Plünderungen in den besetzten Gebieten trugen nicht gerade dazu bei, die Stimmung der Bevölkerung zugunsten der dänischen Okkupanten zu beeinflussen.

Der Vorstoß der 6000 norwegischen Bauernsoldaten ins Götaland war ein vollständiges Fiasko. Später wurde dieser Fehlschlag mit Motivationsproblemen der Norweger erklärt ${ }^{15}$. Diese Probleme hatten norwegische Bauern allerdings nicht, als es darum ging, 300 schottische Söldner auf ihrem Weg durch das Gudbrandsdal zu vernichten.

Erst am 3. August 1611 ergab sich die seit mehreren Wochen belagerte Festung Kalmar den dänischen Truppen. Auch die Festungen Älvsborg und Gullberg am Götafluß mußten sich den dänischen Belagerern ergeben. Ingesamt aber war der Kalmarer Krieg nur ein bedingter Erfolg für König Christian IX. Wesentliche Kriegsziele waren nicht erreicht worden. Der Unterhalt und die Versorgung der ausländischen Söldner entwickelte sich zu einem Problem. Unruhe, Ungehorsam und Plünderungen drohten ständig. So war Christian schließlich daran gelegen, die Feindseligkeiten so schnell wie möglich einzustellen. Allerdings war Schweden zu diesem Zeitpunkt ebenfalls in einer äußerst prekären Lage. Das Land befand sich auch mit Polen im Kriegszustand, und das Verhältnis zu Rußland konnte nur als spannungsgeladen bezeichnet werden. Hinzu kam der die schwierige Lage noch verschärfende Regentenwechsel, als am 30. Oktober 1611 Gustav II. Adolf Karl IX. auf den schwedischen Thron folgte ${ }^{16}$. Als am Ende 1613 der Frieden von Knäred unterzeichnet wurde, konnten beide Seiten zufrieden sein ${ }^{17}$. Dänemark-Norwegen mit König Christian IV. an der Spitze hatte seine Vorherrschaft im Ostseeraum erneut erfolgreich verteidigt, wenn auch zum letzten Mal. Schweden war relativ glimpflich davongekommen, auch wenn das Land zum zweiten Mal nach 1570 ein enormes Lösegeld entrichten mußte, um die Feste Älvsborg auszulösen. Und nach der Thronbesteigung von Gustav II. Adolf und der Konsolidierung seiner Macht innerhalb Schwedens war der Weg frei für eine Entwicklung, die schließlich in der schwedischen Großmachtposition ihren Höhepunkt finden sollte. 


\section{Der Schottenzug}

Neben dem oben schon erwähnten schottischen Offizier Sir James Spens wurde nach Ausbruch des Krieges auch der flämische Oberst Johann von Mönnichhofen ${ }^{18}$ vom schwedischen König mit dem Auftrag in die Niederlande entsandt, dort ein Söldnerheer für die Verteidigung Schwedens zu rekrutieren. Mönnichhofen gelang es bis zum 1. Juni 1612, etwa 1200 Soldaten anzuwerben, die auf vier Schiffen von Amsterdam nach Schweden transportiert werden sollten. Weil allerdings dänische Truppen Älvsborg belagerten, war der Seeweg nach Schweden für die angeworbenen Entsatztruppen versperrt, da sowohl die Landschaft Båhuslen als auch Skåne an der schwedischen Südwestküste unter dänisch-norwegischer Kontrolle standen. So blieb für Mönnichhofen und seine Söldnertruppe nur der Weg durch Norwegen nach Schweden.

Unterdessen hatte die schwedische Krone auch Kontakte nach Schottland geknüpft, um dort Söldner für den Krieg anzuwerben. Allerdings wurden diese Anwerbungsversuche von der englischen Krone mit größtem Mißtrauen verfolgt, war doch der regierende englisch-schottische König Jakob I. mit einer Schwester des dänischen Königs verheiratet.

- Oberst Andrew Ramsey, der als schwedischer Anwerber auftrat, wurde zur Strafe des Landes verwiesen. Seinem Bruder, Oberstleutnant Alexander Ramsey, gelang es aber, sich mit rund 350 Söldnern auf den Weg über die Ostsee nach Osten zu machen. Unter seinem Kommando standen die Hauptleute George Hay und George Sinclair mit ihren beiden Kompanien ${ }^{19}$. Genau dieser George Sinclair, der schon auf der eingangs zitierten Grabinschrift erwähnt wird, sollte später einen herausragenden Platz in den norwegischen Sagen und Legenden rund um den Schottenzug einnehmen.

Alexander Ramsey beabsichtigte, sich auf den Shetland-Inseln mit der kleinen Flotte Mönnichhofens zu vereinigen. Das geplante Treffen schlug jedoch fehl, was für die Expedition Ramseys verhängnisvolle Folgen haben sollte, da Mönnichhofen auch den größten Teil der Waffen für alle angeworbenen Söldner mit sich führte ${ }^{20}$. Mönnichhofens Flotte segelte direkt in den Trondheimsfjord und ging schließlich bei der Ortschaft Stjørdal nördlich von Trondheim an Land. Von hier aus zog der Oberst mit seiner Truppe über das Meråkerfjell durch Jemtland bis nach Stockholm, ohne auf irgendeine Art von Widerstand zu stoßen.

Völlig anders verlief dagegen die Expedition Alexander Ramseys. Am 19. oder 20. August 1612 landeten die rund 350 schottischen Söldner bei Klungnes im Romsdalsfjord ${ }^{21}$. Mit der Hilfe von lokalen Führern zog die Truppè durch das Romsdal in das nördliche Gudbrandsdal, Allerdings war die Kunde von der Landung der Schotten schon durch die Täler geeilt. Der Lensmann (Amtmann) von Dovre, Laurits Hage, hatte bei Sel bewaffnete Bauern aus dem nördlichen Gudbrandsdal und aus Vågå zusammengezogen, die die schottischen Söldner aufhalten sollten. Ih-

In schwedischen Quellen wird von Mönnichhofen als "Generalintendant und Chef " (generalintendant og sjef) oder als "Chefintendant « (sjefintendant) bezeichnet. Siehe Michell, Historien (wie Anm. 4), S. 22.

20 Ebd., S. 46.

21 Ebd., S. 49. 
nen zur Hilfe eilten Bauern aus den weiter südlich gelegenen Kirchspielen Ringebu und Fron unter der Führung von Lensmann Peder Randklev. Bei Kringen, drei Kilometer südlich der heutigen Ortschaft Otta gelegen, kam es am 26. August 1612 zum Kampf zwischen den norwegischen Bauern und den schottischen Söldnern. Bei Kringen verengt sich das Tal und genau hier fand der Überfall statt. Im Bericht von Enevold Kruse heißt es dazu: »Und sie [die Bauern, der Verf.] hatten sie eingeschlossen zwischen den Abhängen auf der einen Seite, und einem großen Fluß auf der anderen Seite. [...] Und innerhalb von anderthalb Stunden waren alle erschossen $^{22}$. An dieser Stelle soll noch einmal erwähnt werden, daß die Schotten, abgesehen von einigen leichten Handwaffen und Musketen, unbewaffnet waren ${ }^{23}$. Die meisten Waffen und Ausrüstungsgegenstände für diese Abteilung hatten sich, wie schon erwähnt, an Bord der Schiffe Mönnichhofens befunden.

Einige Schotten versuchten, über den Fluß Gudbrandsdalslågen zu entkommen. Diejenigen, die bei diesem Versuch nicht ertranken, wurden auf dem westlichen Ufer sofort von norwegischen Bauern erschlagen. Rund fünfhundert norwegische Bauern hatten hier bei Kringen über 200 Schotten getötet, bei sechs eigenen Gefallenen und zwölf Verwundeten ${ }^{24}$. Gefangengenommen wurden 134 Schotten, die, so beschlossen die norwegischen Anführer, unter strengster Bewachung nach Süden auf die Festung Akershus verbracht werden sollten. Doch der Gefangenenzug kam nur bis zur Ortschaft Kvam, $15 \mathrm{~km}$ südlich von Kringen gelegen. Hier wurden die Gefangenen in der Nacht vom 26. auf den 27. August in einer Scheune eingesperrt. Aber anstatt am nächsten Morgen weiter in Richtung Süden eskortiert zu werden, wurden bis auf achtzehn alle schottischen Gefangenen buchstäblich abgeschlachtet ${ }^{25}$. Zu den Opfern dieses Massakers gehörten auch die Offiziere George Sinclair und George Hay. Die achtzehn Überlebenden, darunter der Anführer Ramsey sowie drei weitere Offiziere, wurden schließlich doch noch nach Akershus verbracht und dort vom dänischen Statthalter Enevold Kruse verhört. Auf diesen Verhören sowie auf den Erzählungen der beteiligten Bauern aus dem Gudbrandsdal basiert die wichtigste Quelle zum Schottenzug, nämlich der eingangs erwähnte Bericht des Enevold Kruse.

Uber das Schicksal der achtzehn Überlebenden ist folgendes bekannt: die vier überlebenden Offiziere wurden zurück nach Schottland geschickt, wo sie Strafen wegen Landesverrats erwarteten. Die übrigen blieben in Norwegen. Einige ließen sich später als Söldner im dänisch-norwegischen Heer anwerben.

\section{Legenden- und Sagenbildung rund um den Schottenzug}

Einige Jahrzehnte nach dem Schottenzug setzte die Legenden- und Sagenbildung ein. Der Volkskundler Kjell Bondevik hat diese Legendenbildung umfassend ana-

Ebd., S. 207. Der Originaltext lautet: „Och haffde de dennum saa indklembt, emellem Klippen paa den eene Siide och en stor Elff, dicht derhos paa denn anden siide. [...] Och paa halffanden Tiimestiid att giørre, dennum allesammen ihjelschøt." Øvrelid, Skottetoget (wie Anm. 6), S. 11.

24 Ebd., S. 12.

25 Bericht des Statthalters Enevold Kruse vom 17.9.1612, abgedruckt bei Michell, Historien (wie Anm. 4), S. 207 f. 
lysiert ${ }^{26}$. Viele Legenden und Sagen wurden im 18. und insbesondere im 19. Jahrhundert weiter ausgesponnen, schriftlich niedergelegt oder aber auch literarisch verarbeitet. $\mathrm{Zu}$ den populärsten literarischen Arbeiten über den Schottenzug gehört zweifellos die »Sinclairweise (norwegisch: Sinclairvisa), gedichtet von Edvard Storm im Jahre 1782. Das ganze Lied hat neunzehn Strophen, von denen hier aber nur die achte und die neunte wiedergegeben werden sollen, weil sie besonders deutlich die Legendenbildung widerspiegeln:

»Sie schändeten und sie brannten auf ihrem Wege alles nieder, das ganze Volk wurde gekränkt,

die Hilflosigkeit der Alten rührte sie nicht,

sie verspotteten die weinende Witwe.

Das Kind wurde im Schoße der Mutter getötet

so freundlich und mild es auch lächelte.

Aber die Kunde über diesen Jammer und die Not

eilte in die Herzen des Landes ${ }^{27}$.

Glaubt man diesen Versen, dann sind die Schotten marodierend durch das Romsdal und das Gudbrandsdal gezogen. Dafür findet sich allerdings kein Beleg in der Darstellung des Enevold Kruse vom 17. September 1612. In seinem zweiten Bericht vom 3. Oktober 1613 schreibt er sogar, daß »die Schotten weder gebrandschatzt, noch gemordet oder andere Zerstörungen angerichtet hatten ${ }^{28}$.

Im 19. Jahrhundert taucht eine neue Version von den Kämpfen bei Kringen auf. Es war der bekannte norwegische Dichter Henrik Wergeland, der sie in seiner norwegischen Geschichte aus dem Jahre 1834 präsentierte ${ }^{29}$. Danach sollen die norwegischen Bauernsoldaten behauene Baumstämme sowie große Steine von den Hängen auf die Schotten gerollt haben. Auch für diese neue Variante findet sich kein Beleg in den Quellen.

Eine weitere Legende besagt, daß die Frau des Hauptmann Sinclair mit einem Neugeborenen am Zug durch die Täler teilgenommen haben soll. Sowohl Frau als auch Kind sollen bei Kringen getötet worden sein. Zwar war es in jener Zeit durchaus üblich, daß Frauen die Söldnerheere begleiteten, aber in den seltensten Fällen waren es die Ehefrauen. Auch für diese Legende finden sich keine Hinweise in den Quellen.

Eine der interessantesten Sagen, die um den Schottenzug entstanden ist, handelt von dem Mädchen Pillar-Guri, das von einem Berggipfel mit einem Horn die im Hinterhalt bei Kringen lauernden Bauernsoldaten vom Herannahen der Schotten gewarnt haben soll. Weder für diese Tat noch für die Existenz des Mädchens gibt es Belege in den Quellen. Die erste Erwähnung dieser Mädchengestalt findet sich in den von Andreas Faye 1833 veröffentlichten Norwegischen Sagen ${ }^{30}$. Seit-

26 Kjell Bondevik, Studiar i norsk segnhistorie, Oslo 1948, S. 9-81.

27 Alle neunzehn Strophen sind wiedergegeben bei Michell, S. 81-84. Die Strophen acht und neun stehen auf der S. 82. Der Originaltext lautet: "De skjendte og brente der de drog frem,/ all folkerett monne de krenke,/ oldingens avmakt rørte dem ei, / de spottet den gråtende enke.// Barnet blev dræpt i moderens skjød/ så blidt og så mildt det enn smilte./ Men rygtet om denne jammer og nød/ til hjertet av landet ilte.«

28 Zit. nach Øvrelid, Skottetoget, S. 14. Der Originaltext lautet: "skottene aldeles intet har brent, myrdet eller ødelagt."

29 Bondevik, Studiar (wie Anm. 26), S. 39.

30 Andreas Faye, Norske sagn, Arendal 1833. 
dem geistert diese Gestalt durch die regionale Sagenwelt. Sogar ein Berggipfel in der Gemeinde Sel trägt den Namen Pillar-Guri-toppen (deutsch: Pillar-Guri-Spitze).

Im Jahre 1789 wurde das Gudbrandsdal von einer gewaltigen Überschwemmungskatastrophe heimgesucht. Dabei soll der alte Gedenkstein auf dem Massengrab der bei Kvam massakrierten Schotten von den Fluten weggespült worden $\operatorname{sein}^{31}$. Auf dem neu errichteten Stein, dessen Inschrift eingangs zitiert worden ist, taucht zum ersten Mal das Zahlenverhälțis von 900 schottischen Söldnern gegen weniger als 300 norwegische Bauern auf. Wiederum war es Andreas Faye, der diese Zahlen in seinem Lehrbuch zur norwegischen Geschichte ungeprüft übernahm ${ }^{32}$. Weiter wird George Sinclair in dieser Grabinschrift zum Oberst erhoben, und es entsteht der Eindruck, daß er der Anführer der schottischen Söldner gewesen sei, was er aber nachweislich nicht war.

Bis heute hat das nationale und regionale Interesse am Schottenzug nicht nachgelassen. Immer wieder werden die Ereignisse des Jahres 1612 in Büchern über das Tal, in regionalen Jahrbüchern sowie in der Lokalpresse aufgegriffen. Oft leben in diesen Darstellungen die Legenden weiter, die rund um den Schottenzug entstanden sind. Ein Beispiel dafür bietet der Artikel »Das Gudbrandsdal in der norwegischen Sage« (norwegisch: Gudbrandsdalen i Norsk Soge) des Heimatforschers Einar Hovdhaugen aus dem Jahre $1974^{33}$. Nach Hovdhaugen ist der Gebrauch von Baumstämmen und von Steinblöcken zum Aufhalten der Söldner bei Kringen als historische Tatsache anzusehen ${ }^{34}$. Allerdings äußert er vorsichtige Kritik an dem Massaker bei Kvam, das er "als keinen ehrenvollen Abschluß « ${ }^{35}$ der Ereignisse bezeichnet.

Sehr kritisch sind die Darstellungen von Rolf Rasch-Engh zum Schottenzug zu betrachten, die zwar spannend und unterhaltend zu lesen sind, aber mit den historischen Fakten nur noch wenig zu tun haben ${ }^{36}$. Nicht nur, daß er die rund um den Schottenzug entstandenen Sagen und Legenden völlig unkritisch übernimmt, nein, er strickt an einer weiteren Legende, die in dieser Version vorher in der Literatur noch nicht aufgetaucht ist. In einem Artikel im Jahrbuch für das Gudbrandsdal 1994 schreibt Rasch-Engh, daß die Vorfahren des George Sinclair als norwegische Jarle, ernannt vom norwegischen König Håkon VI, im Jahre 1379 auf die OrkneyInseln kamen, das heißt Sinclair kam als Soldat, wenn auch als feindlicher, zurück in das Land seiner Vorväter ${ }^{37}$. Nicht einen einzigen schriftlichen Beleg präsentiert Rasch-Engh für seine »Geschichte«. Er wiederholt diese Behauptungen in einer Broschüre aus dem Jahre $1996^{38}$, in der er überdies in geradezu peinlicher Weise den ungleichen Kampf bei Kringen, von norwegischen Autoren übrigens oft als "Schlacht bei Kringen« bezeichnet, verteidigt, als er schreibt:

Zur Überschwemmungskatastrophe und den Folgen für das Gudbrandsdal siehe Ragnar Øvrelid, Historia om Fron fram til 1940, Tretten 1987, S. 73 f.

Andreas Faye, Norges Historie, Christiania 1831.

Einar Hovdhaugen, Gudbrandsdalen i Norsk Soge, in: Bygd og By i Norge. Gudbrandsdalen. Utg.: Knut Ramberg, Oslo 1974, S. 102-140, hier besonders die S. 122-124. Ebd., S. 124.

Ebd., S. 124. Der Originaltext lautet: "Det var ingen ærerik avslutning.«

Rolf Rasch-Engh, Sinclair-soga-ennå ein gong, in: Arbok for Gudbrandsdalen 1994, S. 185-200; ders., Herr Sinclair som dro over salten hav, Brattvåg 1996.

Rolf Rasch-Engh, Sinclair-soga (wie Anm. 36), S. $186 \mathrm{f}$.

Rasch-Engh, Herr Sinclair (wie Anm. 36), S. 2. 
»Das Großartige bei Kringen im Jahre 1612 war, daß die Bauern gegen einen eindringenden Feind zu den Waffen griffen, sowie die Durchführung des Kampfes. Die Wahl des Kampfplatzes, die Planung und die Führung waren absolute Spitzenklasse. Professionelle Soldaten hätten es nicht besser machen können. Die Führer der Bauern mußten einen ungewöhnlich guten Zugriff auf ihre Leute gehabt haben, und diese [die Bauernsoldaten, der Verf.] haben eine ungewöhnliche Disziplin gezeigt ${ }^{39}$.«

Für das Gemetzel am nächsten Tag findet Rasch-Engh gleich mehrere Entschuldigungen. Danach sollen Gerüchte über ein Massaker schwedischer Söldner an Norwegern, Alkoholmißbrauch sowie die Tatsache, daß man sich in der besten Erntezeit befand und den langen Weg und die damit verbundene Abwesenheit von den heimatlichen Höfen scheute, zu dem Morden geführt haben ${ }^{40}$. Es ist durchaus denkbar, daß exzessiver Alkoholmißbrauch unter den Wachen schließlich zu diesem Blutbad geführt haben kann.

$\mathrm{Zu}$ den seriösen Forschungsarbeiten der letzten Jahre zählen, besonders von der sorgfältigen Quellenkritik her gesehen, die schon zitierten Darstellungen des Historikers Ragnar Øvrelid ${ }^{41}$.

Betrachten wir abschließend die Sagen- und Legendenbildung rund um den Schottenzug, so ist es nicht verwunderlich, daß die meisten im 19. Jahrhundert schriftlich riedergelegt und dabei oft weiter ausgesponnen wurden. Im Jahre 1814 mußte Dänemark im Kieler Frieden Norwegen an Schweden abtreten. Vergeblich versuchte Norwegen, sich einer Union mit Schweden zu widersetzen. In einer kurzen, nur wenige Monate dauernden Phase der Unabhängigkeit, wurde eine Verfassung für das Land ausgearbeitet, die für ihre Zeit richtungweisend war. Als dann im 19. Jahrhundert der norwegische Nationalismus zu blühen begann, der schließlich im Jahre 1905 zur Auflösung der ungeliebten Union mit Schweden führen sollte, gab es einen enormen Bedarf für Mythen und Helden, die sich, angeblich heroisch, fremden Eindringlingen in den Weg gestellt hatten.

Wie wir gesehen haben, nimmt der Schottenzug einen gewichtigen Platz in der Regional- und Lokalgeschichte ein. Heute liegen die beiden "Hauptkriegsschauplätze« des Schottenzuges; nämlich Kringen und die Ortschaft Kvam, Schauplatz des Massakers an den gefangengenommenen Schotten, in zwei verschiedenen Gemeinden. Kringen gehört zur Gemeine Sel mit dem Hauptort Otta. Die Ortschaft Kvam dagegen gehört zur Gemeinde Nord-Fron mit dem Hauptort Vinstra.

Im Jahre 1995 ist in Kvam ein Kriegsgeschichtliches Museum eröffnet worden, das sich der Kriegsgeschichte des Gudbrandsdals annehmen möchte. Da nimmt natürlich der Schottenzug, neben der deutschen Okkupationszeit von 1940 bis 1945, einen herausragenden Platz ein. Die Pläne der Museumsleitung gehen dahin, die Ausstellung über den Schottenzug nach und nach deutlich zu erweitern. Diese Absicht wird allerdings von der Gemeinde Sel nicht unterstützt. Dort hütet die Gemeinde eifersüchtig Gegenstände und Überreste, die angeblich dem Schottenzug zuzuordnen sind. Ziel der Gemeinde Sel ist es nämlich, auch ein Museum einzu-

Ebd., S. 11 f. Der Originaltext lautet: "Det store ved Kringen i 1612 var at bøndene grep til vápen mot en inntrengende fiende, og selve gjennomføringen av kampen. Valget av kampplass, planlegging og ledelse er helt forsteklasses. Profesjonelle soldater kunne ikke ha gjort det bedre. Førerne for bondene må ha hatt et usedvanlig godt grep på sine folk, og disse har visst en utrolig disiplin.«

40 Ebd., S. 13.

${ }_{41}$ Zum Beispiel Øvrelid, Skottetoget (wie Anm. 6), S. 9-17. 
richten, das den Schottenzug darstellen soll. Im Rahmen dieser Konkurrenz um das 'Erbe tauchen auch in kontinuierlichen Abständen Berichte über angebliche neue Quellenfunde zum Schottenzug in der örtlichen Presse auf, wie folgendes Beispiel belegt: Am 30. August 1997 präsentierte eine hohe Verwaltungsbeamtin der Gemeinde Sel bei einem offiziellen Pressetermin ein im Keller des Rathauses von Otta gefundenes Paket aus dem Jahre 1912, das angeblich eine "geheime Kriegsgeschichte« der Kämpfe bei Kringen enthalten sollte ${ }^{42}$. Auf dem Paket ist folgender Text zu lesen: »Kringengedenkstein und Kringenfest 26. August 1912. Kann im Jahre 2012 geöffnet werden. Gez. Joh. Nygaard ${ }^{43}$."

Am 8. September 1997 meldete sich darauf in der Lokalzeitung mit dem damals 86jährigen ehemaligen Schulrektor Olav I. Haugen aus der Nachbargemeinde Vågå ein historisch Interessierter, der die Darstellung der Gemeindebeamtin stark in Zweifel zog ${ }^{44}$. Als junger Mann will Olav I. Haugen im Jahre 1927 oder 1928 dabeigewesen sein, als der Lehrer und ehemalige Bürgermeister Joh. Nygaard das ominöse Paket in einer offiziellen Zeremonie an seinen Nachfolger, den Bürgermeister Arthur Jansen überreichte. Wörtlich sagte Olav Haugen am 8. September 1997 in der Zeitung über den Inhalt des Pakets: "Ich glaube nicht, daß neue Erkenntnisse über die Schlacht bei Kringen auftauchen, sondern nur über die Feierlichkeiten von 1912.45 . « Wie Olav Haugen bin auch ich überzeugt, daß dieses Paket keine neuen wesentlichen Erkenntnisse ans Tageslicht bringen wird, und schon gar keine neuen, verwertbaren Quellen. Darauf weist meiner Meinung nach schon die Paketaufschrift hin. Hier ist nur die Rede von einem Gedenkstein sowie einem Fest, das an das dreihundertjährige Jubiläum der Kämpfe bei Kringen erinnern sollte. Und doch wird es im Jahre 2012 sicherlich Lokal- und Hobbyhistoriker geben, die mit Spannung der offiziellen Öffnung des Pakets entgegenfiebern werden, in der Hoffnung, vielleicht doch noch neue, unbekannte Quellen zum Schottenzug ans Tageslicht zu fördern.

Ein weiterer Beleg für die Vereinnahmung von Sagengestalten aus dem Schottenzug ïst die Stiftung des Pillar-Guri-Preises in der Gemeinde Sel. Seit 1996 wird dieser Preis an Persönlichkeiten verliehen, die sich in besonderer Weise um die Kultur in der Gemeinde Sel sowie im nördlichen Gudbrandsdal verdient gemacht haben.

Bei der Stiftung dieses Preises war eine spürbare Rivalität mit der Gemeinde Nord-Fron nicht zu übersehen. In dieser Gemeinde wird jährlich Anfang August im Rahmen der angesehenen Peer-Gynt-Festspiele die Peer-Gynt-Statue an eine bekannte norwegische Persönlichkeit verliehen, die sich in besonderer Weise um das Ansehen des Landes im Ausland verdient gemacht hat ${ }^{46}$.

Neben dem kriegsgeschichtlichen Museum in Kvam gibt es in der Ortschaft noch ein Gasthaus mit dem Namen "Vertshuset Sinclair» (Sinclairs Gasthaus), des-

42 Gudbrandsdølen Dagningen, 30.8.1997. Der Artikel war folgendermaßen betitelt: »Heimliche Kriegsgeschichte gefunden « (norwegisch: Fant hemmelig krigshistorie).

43 Ebd. Der Originaltext auf dem Paket lautet: »Kringenstøtten og Kringenfesten 26 de august 1912. Kann aabnes aar 2012. Joh. Nygaard. « Joh. Nygaard war Lehrer und einige Jahre Bürgermeister der Gemeinde Sel, unter anderem im Jahre 1912.

44 Gudbrandsdølen Dagningen, 8.9.1997.

45 Ebd. Der Originaltext lautet: $»$ Eg trur ikkje det kjem fram nye opplysningarn om Slaget ved Kringen, men om den festlege feiringa i 1912."

46 Zu den Preisträgern der Peer-Gynt-Statue zählen zum Beispiel die ehemalige norwegische Staatsministerin Gro Harlem Brundtland, der weltbekannte norwegische Archäologe Thor Heyerdahl sowie der Schriftsteller Jostein Gaarder. 
sen Besitzer sich der Sammlung von Gegenständen aus der Zeit rund um den Schottenzug verschrieben hat. Eines der wertvollsten Stücke dieser Privatsammlung ist eine schottische Muskete, die von den siegreichen Bauern zusammen mit vier anderen Musketen nach dem Massaker bei Kvam mit den wenigen Überlebenden beim dänischen Statthalter Enevold Kruse abgeliefert worden war. Viele Jahre lagerten diese Waffen im Arsenal auf der Festung Akershus, bis dieses eine Exemplar über verschiedene Wege in die Hände des Gastwirtes bei Kvam gelangt ist. Daneben hat er noch eine Reihe von Bildern, die den Schottenzug darstellen, sowie Ausrüstungsgegenstände aus dieser Zeit gesammelt.

Das blutige Geschehen im Gudbrandsdal hatten keinerlei Auswirkung auf den Ausgang des Kalmarer Krieges. Allerdings sorgten die Ereignisse im Gudbrandsdal mit dafür, daß Norwegen eine neue Heerordnung erhielt, die 1628 verabschiedet wurde. Diese Ordnung sah vor, fünf Regimenter (6240 Soldaten) aufzustellen, die im Kriegsfall zusammengezogen werden konnten ${ }^{47}$. Auf diese Weise sollten sicherlich auch wilde Bauernhaufen, die allerdings bei Kringen und Kvam ihre »Effektivität« bewiesen hatten, kontrolliert werden.

47 Norges Historie, Bd 6 (wie Anm. 10), S. 410. Siehe auch Thuesen, Norges Historie i Árstall (wie Anm. 13), S. 141. 


\section{Oldenbourg}

\section{Jahrbuch für Europäische Geschichte}

Band 1: 2000

Das Jahrbuch für Europäische Geschichte stellt sich vor

als Forum für Experten aus den europäischen Ländern, die zu herausragenden Themen, Problemen und Grundsatzfragen der europäischen Geschichte Stellung nehmen

als Podium für die ausgewiesenen Kenner aus Europa und Übersee, die Forschungstrends und Forschungsergebnisse aus ihren Ländern vorstellen

als Arena für Originalbeiträge vorwiegend jüngerer Wissenschaftler.

Im Aufsatzteil erscheinen wissenschaftliche Arbeiten, der Informationsteil bietet Forschungsberichte zu Themen der europäischen Geschichte und zur europabezogenen Grundlagenforschung, Berichte aus der Arbeit der Europainstitute und Europaliteratur.

Das Jahrbuch erscheint ab Frühjahr 2000 in einem Umfang von ca. 240 Seiten.

Der erste Band des Jahrbuchs hat das Schwerpunktthema »Europa - eine Bilanz des 20. Jahrhunderts « und enthält Beiträge von Curt Gasteyger, Heinz-Gerhard Haupt, Wilfried Loth, Hans Mommsen, Hans-Ulrich Thamer und Hermann van der Wee.

Die Forschungsberichte sind dem Westfälischen Frieden 1648-1998 (Johannes Arndt) und den Revolutionen von 1848 (Dieter Hein) gewidmet. Georg Kreis berichtet über die Arbeit des Europa-Instituts an der Universität Basel.

Ihre Bestellung richten Sie bitte an Ihren Fachbuchhändler oder direkt an: verkauf-f@verlag.oldenbourg.de
Schwerpunktthemen der kommenden Bấnde

-Europäisches Recht in Übersee

-Beziehungsgeschichten europäischer Nachbarn -Währungsunionen

-Europäische Integration durch Kommunikation und Verkehr

-Der Europa-Diskurs an der europäischen Peripherie.

2000. VII, 239 Seiten,

Fortsetzungspreis DM 64,Einzelpreis DM 78,ISBN 3-486-56464-1

Herausgegeben am Institut für Europäische Geschichte von Heinz Duchhardt in Verbindung mit Wlodzimierz Borodziej, Peter Burke, Ferenc Glatz, Georg Kreis, Pierangelo Schiera, Winfried Schulze 